\title{
The Birth, Growth, Death and Rejuvenation of Software Maintenance Communities
}

\author{
Qiong Feng, Yuanfang Cai \\ Drexel University \\ Philadelphia, PA \\ \{qf28,yc349\}@drexel.edu
}

\author{
Rick Kazman \\ University of Hawaii \& SEI/CMU \\ Honolulu, HI \\ kazman@hawaii.edu
}

\author{
Ran Mo \\ Drexel University \\ Philadelphia, PA \\ rm859@drexel.edu
}

\begin{abstract}
Background: Though much research has been conducted to investigate software maintenance activities, there has been little work charactering maintenance files as a community and exploring the evolution of this community. Aims: The goal of our research is to identify maintenance communities and monitor their evolutionbirth, growth, death and rejuvenation. Method: In this paper, we leveraged a social community detection algorithm-clique prelocation method (CPM)-to identify file communities. Then we implemented an algorithm to detect new communities, active communities, inactive communities and reactivated communities by cumulatively detecting and constantly comparing communities in time sequences. Results: Based on our analysis of 14 open-source projects, we found that new communities are mostly caused by bug and improvement issues. An active community can be vigorous, on and off, through the entire life of a system, and so does an inactive community. In addition, an inactive community can be reactivated again, mostly through bug issues. Conclusions: These findings add to our understanding of software maintenance communities and help us identify the most expensive maintenance spots by identifying constantly active communities.
\end{abstract}

\section{CCS CONCEPTS}

- Software and its engineering $\rightarrow$ Software evolution;

\section{KEYWORDS}

Software Evolution, Maintenance Communities

\section{ACM Reference Format:}

Qiong Feng, Yuanfang Cai, Rick Kazman, and Ran Mo. 2018. The Birth, Growth, Death and Rejuvenation of Software Maintenance Communities. In ACM / IEEE International Symposium on Empirical Software Engineering and Measurement (ESEM) (ESEM '18), October 11-12, 2018, Oulu, Finland. ACM, New York, NY, USA, 10 pages. https://doi.org/10.1145/3239235.3239246

\section{INTRODUCTION}

Much research $[15,17,21,24]$ has been conducted to investigate software maintenance activities within groups of files. Xiao et al. [24]

Permission to make digital or hard copies of all or part of this work for personal or classroom use is granted without fee provided that copies are not made or distributed for profit or commercial advantage and that copies bear this notice and the full citation on the first page. Copyrights for components of this work owned by others than ACM must be honored. Abstracting with credit is permitted. To copy otherwise, or republish, to post on servers or to redistribute to lists, requires prior specific permission and/or a fee. Request permissions from permissions@acm.org.

ESEM '18, October 11-12, 2018, Oulu, Finland

(c) 2018 Association for Computing Machinery.

ACM ISBN 978-1-4503-5823-1/18/10 ..\$15.00

https://doi.org/10.1145/3239235.3239246 proposed a model called DRSpace, which is a set of architecturalconnected files. They claimed that a few DRSpaces can capture most buggy files in a project, and can furthermore reveal the structural relations among these buggy files. Silva et al. [21] studied co-changed files in software maintenance and classified them into 3 patterns: Encapsulated, Crosscutting, and Octopus. Based on these patterns, they further investigated developers' perceptions of cochange clusters and detected design anomalies reflected by these co-change patterns. Negara [17] proposed a new algorithm to mine previously unknown patterns from fine-grained sequences of code changes; they summarized and evaluated 10 popular types of transformations. Jiang et el [15] presented an approach to group and aggregate relevant code changes into six types of trajectory patterns and detected underlying rules with these patterns. While patterns in a group of either structurally-related or co-changing files have been extracted, there is little study characterizing co-changing files as a community and exploring the evolution of this community.

In this paper, we leverage a social community detection algorithmcalled clique prelocation method $(C P M)[8,19]$-to identify file communities in maintenance. The file communities detected by $C P M$ have two characteristics: (1) files in the same community co-change with each other very frequently; (2) files in the same community are more likely to be located in the same packages. In other words, files in the same community are more likely to share some key characteristics and to be changed together. These file communities, we claim, should be analyzed as a group, to understand a system's evolution.

We have implemented an algorithm to detect new communities, active growing communities, active closed communities, inactive communities, and reactivated communities. We do this by detecting and constantly comparing communities in a time sequence. We treat each issue as a unit and each issue's resolution time as a time stamp. For example, in our study, the Avro project has 689 issues from $i 1$ to $i 689$ so we have 689 time stamps from $t 1$ to $t 689$. We run the $C P M$ algorithm to cumulatively detect communities at each time stamp. For example, at $t 1$, we detect communities using $i 1$ 's files; at $t 2$, we detect communities using i1's files and $i 2$ 's files, etc. A new community is deemed to be born at $t_{x+1}$ when this community exists at $t_{x+1}$ but does not exist at $t_{x}$. An active open community at $t_{x+1}$ is a community whose size is growing from time $t_{x}$ to time $t_{x+1}$. An active closed community at time $t_{x+1}$ is a community in which files are modified in issue $i_{x+1}$ but no new files are subsequently added to this community. Since its last activity, if files in a community have no activities and this community is not growing for a period of time $(\Delta t)$, at a settable threshold, we call this an inactive community. If an inactive community becomes alive again (either new files are added or existing files are modified), we call this a reactivated community. 
Based on our analysis of 14 open-source projects, we found that the majority ( $74.5 \%$ to $92.5 \%$ ) of maintenance files can be clustered into communities. So community evolution can reflect most of a system's evolution. New communities are typically formed by bug and improvement issues. An existing community can grow, on and off, through the entire life of a system. An inactive community, usually containing a small set of files, can start as early as the project's beginning and last till the final time stamp in our analysis. And an inactive community can be activated again, typically through bug issues.

The contributions of this paper are as follows:

(1) Our work is the first empirical study of software file maintenance communities and their evolution by using a social community detection algorithm $(C P M)$.

(2) We implemented an algorithm to monitor the life cycle of maintenance communities: their birth, growth, death and rejuvenation. This algorithm is general and can be applied to detect the life cycle of any sets of files, and not just file community detection.

(3) Our algorithm can detect the most expensive growing maintenance communities, where file interactions can be visualized. Developers can look into these problematic communities and try to find a refactoring solution.

The rest of the paper is organized as follows. Section 2 lists work related to our research. Section 3 presents how communities are extracted and communities' characteristics. Section 4 defines the stage of a community's life cycle. Section 5 states the 5 research questions and section 6 demonstrate the experiments and the answers to these research questions. Section 7 discusses the results and our future work. Finally this paper concludes by section 8 .

\section{RELATED WORK}

\subsection{Software Communities}

Software communities have been extensively studied [1, 2, 4, 9, $10,12,13,22]$. Barcellini et al. [1,2] studied how members of OSS communities, and especially users, participate in the design process and to identify whether or not some participants are the key to connect the user and the developer communities. Ducheneaut [9] explored the communications in the email and code databases of OSS and characterized successful participants. Fang and Neufeld [10] conducted qualitative analyses and concluded that sustained participations are not caused by the initial condition to participate, but due to situated learning and identity construction behaviors. Bird et al. [4] extracted and studied latent sub-communities from the email social network. Their results show that subcommunities do indeed spontaneously arise as the projects evolve. Heller et al. [13] analyzed user profiles and repository metadata from the GitHub source code hosting service and summarized 3 patterns based on developer relationships, social connectivity and influence among cities, and variation in project specific contribution styles (e.g., centralized vs. distributed). Hahn et al. [12] explored how developers choose to contribute to newly initiated OSS projects. Their results claimed that a project is more likely to attract a developer if the project's initiator has strong collaborative ties with the developer. Tamburri et al. [22] described a set of community smells that can plague an architecture, as well as a set of mitigations for each.
All of the above studies have provided valuable insights on developers or users' communities, however, none of these studies have considered software source files as members in a community. Files co-change with each other in the project's revision history, just as developers communicate with each other via email. In this paper, we treat each file as an element of a maintenance community to learn how files interact with each other in a community and how maintenance communities communicated with each other.

\subsection{Software Evolution}

Software evolution, especially software evolution modeling, has attracted substantial attention. Chapin et al. [6] proposed a method to classify evolution types based on a semi-hierarchical manner of the change in 4 different aspects from documentation to functionality. Godfrey et al. [11] compared software evolution with biological evolution, and examined how software evolves in response to environmental pressure and emergent design. Tu et al. [23] claimed the evolution of the Linux kernel at the system level and within the major subsystems is fast, and discussed why they think Linux continues to exhibit such strong growth. Robles at al. [20] studied the evolution of a Linux distribution, Debian, with respect to its overall size, use of programming languages, maintenance of packages, and file sizes over seven years.

Achitectural evolution has also been explored [5, 25, 26]. Chaikalis et al. [5] incorporated structural information and domain information, such as the creation of relations among existing and new classes and the removal of edges, into a network-based prediction model. 10 open-source projects were used to evaluate this model and they showed that their derived models can provide insight into the future trends and can potentially form the basis for eliciting improved or novel laws of software evolution. Zimmermann [26] examines architectural refactoring as an evolution technique that revisits architectural decisions and identifies related design, implementation, and documentation tasks. Xiao et al. [25] also studied software evolution in terms of architectural debt. They proposed 4 types of architectural issues and showed how these issues evolve into architectural debt over time.

The above studies on software evolution focus on the evolution of software architecture, knowledge bases, and so forth. Our study is complementary to these studies, by investigating software evolution through maintenance file communities.

\subsection{Co-change Patterns in Maintenance}

Much prior research $[15,17,18,21]$ has studied co-change patterns in maintenance. Silva et al. [21] studied co-changing files software maintenance and classified them into 3 patterns: Encapsulated, Crosscutting, and Octopus. Based on these patterns, they further investigated developers' perception of co-change clusters and detected design anomalies reflected by these co-change patterns. Negara et el. [17] proposed an algorithm to mine previously unknown patterns from fine-grained sequences of code changes; they then summarized and evaluated 10 popular types of transformations. Jiang et el. [15] presented an approach to group and aggregate relevant code changes as six types of trajectory patterns and detected underlying rules with these patterns. Nguyen et al. [18] studied 
Table 1: Subjects

\begin{tabular}{|l|c|r|r|r|}
\hline Subject & Start Time & \# Files & \# Commits & \# Issues \\
\hline Avro & $2009-04$ & 1458 & 1560 & 689 \\
\hline Camel & $2007-03$ & 24466 & 28899 & 6396 \\
\hline Cassandra & $2009-03$ & 3027 & 23026 & 5018 \\
\hline Cxf & $2008-04$ & 10905 & 13135 & 3321 \\
\hline Derby & $2004-08$ & 4430 & 8088 & 2069 \\
\hline Hadoop & $2009-05$ & 10027 & 16103 & 2457 \\
\hline Hbase & $2007-04$ & 4436 & 13564 & 6516 \\
\hline Mahout & $2008-01$ & 2328 & 3799 & 694 \\
\hline Openjpa & $2006-05$ & 5211 & 4820 & 1188 \\
\hline Pdfbox & $2008-02$ & 1672 & 6239 & 1771 \\
\hline Pig & $2009-03$ & 2452 & 3076 & 1639 \\
\hline Tika & $2007-03$ & 2062 & 3794 & 1033 \\
\hline Wicket & $2004-09$ & 5619 & 19846 & 3146 \\
\hline Zookeeper & $2008-05$ & 1192 & 1557 & 786 \\
\hline
\end{tabular}

repetitive changes within and across projects by comparing old and new AST subtrees.

While these co-change studies have successfully captured cochange patterns among multiple files with co-change relations in maintenance, none of these studies are charactering co-change maintenance files as a community and exploring the evolution of this community. These patterns can change or disappear as software evolves. Our paper investigates a maintenance community's life cycle, including how it is born, grows, achieves stability and eventually becomes inactive.

\section{MAINTENANCE COMMUNITIES}

\subsection{Subjects}

For our study we selected 14 open source projects from Apache, as evaluation subjects. These projects differ in terms of size, domain, and length of evolution history. They range in domains, from a distributed database (Hadoop) to a content analysis toolkit (Tika). Table 1 shows the "demographic" information for each project. We studied each project from the start dates listed in the Start Date column of the table until 2017-07-07. Their ages vary from 13 years old (Wicket) to 8 years old (Hadoop).

The \#Files column lists the number of files found in their latest version, varying from 1458 files in Avro to 24466 files in Camel. The number of commits recorded in their evolution history, that is, the number of revisions made to each project, ranges from 1560 in Avro to 28899 in Camel. Column \#Issues lists the number of bug issues recorded in their respective JIRA databases.

\subsection{Extraction of Maintenance Communities}

In this section, we describe how software maintenance communities are extracted. The data in the paper can be accessed via Zenodo (https://doi.org/10.5281/zenodo.1321253). Given a project and a time range, we conduct the following steps.

Step 1: Extract issue keys from its JIRA issue tracking system.

Step 2: Find a set of files for each issue in the revision log where the commit message matches the issue key. In this step, all test files are filtered out as we focus our investigation on just source files. In this step, we also filter out issues that modified more than 10 source files, to reduce the noise caused by a large set of files (due to changes in formatting, refactorings, etc). We choose 10 because the issues with fewer than 10 files cover around $95 \%$ of all issues.

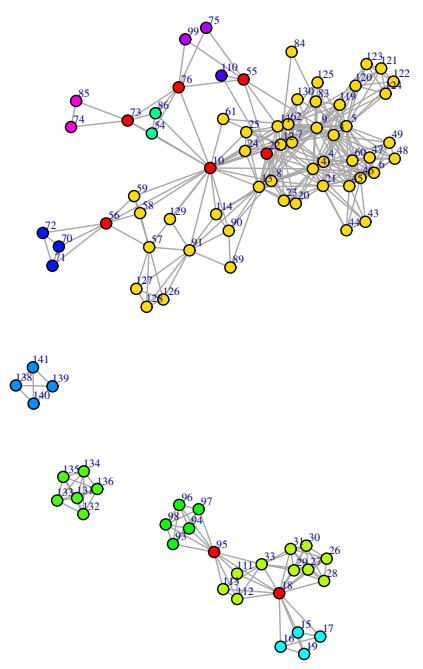

$0^{52}$
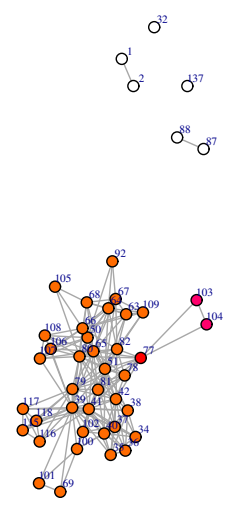

Figure 1: Communities Formed by the First 100 Avro Issues

Step 3: Extract co-change edges for each issue. If two files are in the same file set and modified for the same issue, then we two form an edge between these files. We extracted all co-change edges in the system maintenance history.

Step 4. Apply the clique prelocation method $(C P M)[8,19]$ to detect software maintenance communities. "The key features of the communities obtained by $C P M$ are that (i) their members can be reached through well connected subsets of nodes, and (ii) the communities may overlap(share nodes with each other)." We choose 3-cliques as a parameter in CPM detection, which means that any file is co-changing with at least two other files in this community. Then we use R scripts to visualize the detected communities.

Figure 1 shows detected maintenance communities from the first 100 issues in Avro. One node represents a distinct file and the nodes in the same color means that these nodes belong to the same community. If a node's color is red, it means that this node belongs to multiple communities. If a node's color is white, it means that this node does not belong to any community. If a file does not cochange with any other files and then it can not form an edge; in this case this file should be isolated in the community representation. Figure 1 shows that 12 communities are formed by these 100 issue fixes. The largest community is shown in yellow. One of the red nodes 95 belongs to two communities as it co-changes with nodes (93-94,96-98) and with nodes (111-113 and 33). There are 6 white nodes without communities (1, 2, 52, 53, 87 and 88), though some nodes co-changed with other other nodes. 2 files (32 and 137) are isolated and they are changing independently.

\subsection{Characteristics of Maintenance Communities}

Bonding in communities: Suppose $C$ is a superset of communities, containing all communities from $c_{m}$ to $c_{n}$ in a system. We calculate the average edge weight inside a community $W_{c}$ and compare it with the average edge inter community weight $W_{i c}$. Suppose $e^{e d g e_{m}}$ is the number of edges between any two files and file $_{m}$ is 
the number of files in the community $c_{m}$. The average edge weight in the community $c_{m}$ is $W_{c_{m}}=e d g e_{m} /\left(\right.$ file $_{m} \times\left(\right.$ file $\left.\left._{m}-1\right) / 2\right)$. Then, we calculate the average edge weight inside one community $W_{c}$ for all communities $(C)$ by $\sum_{c_{m} \in C} W_{c_{m}} / \operatorname{Size}(C)$.

Similarly, edge $m n$ is the number of edges between a file in community $c_{m}$ and a file in community $c_{n}$. The average edge inter community weight $W_{i c}$ is $\frac{\sum_{c_{m} \neq c_{n}} e d g e_{m n}}{\operatorname{Size}(C) \times(\operatorname{Size}(C)-1) / 2}$.

From figure 1, it is obvious that $W_{c}$ is significantly larger than $W_{i c}$, which means that the bonding in the same community is much tighter than between different communities. This indicates that files in same community tend to change together in maintenance activities. Table 2 shows $W_{c}$ and $W_{i c}$ of the 14 subject projects. The ratio of $W_{c}$ to $W_{i c}$ ranges from 6.1 to 130.4 , with an average of 41.1, meaning that a file co-changes with another file in the same community 41.1 times more often, on average, than a file co-changes with a file in another community. This justifies our community definition.

Location in communities We define the PathDistance of two files based on the packages they belong to. If two files are located in the same package, then their distance is 0 . Given two files, $f_{i}$ and $f_{j}$, we define $\operatorname{Dir}_{c}$ as the common directories shared by both files, and model their full paths as:

$\operatorname{Path}\left(f_{i}\right)=$ ProjRoot $/ \operatorname{Dir}_{c} / \operatorname{Dir}_{A 1} / \operatorname{Dir}_{A 2} / \ldots / \operatorname{Dir}_{A n} / f_{i}$

$\operatorname{Path}\left(f_{j}\right)=$ ProjRoot $/ \operatorname{Dir}_{c} / \operatorname{Dir}_{B 1} / \operatorname{Dir}_{B 2} / \ldots / \operatorname{Dir}_{B m} / f_{j}$

We define the distance as: PathDistance $\left(f_{i}, f_{j}\right)=n+m$. Modeling all the files involved in a community, $c_{m}$, as a set, FileSets and $f_{i}, f_{j} \in$ FileSets, we can compute the average path distance of the file set AvgPathDistance $\left(c_{m}\right)$ as follows:

$$
\sum_{i \neq j} \text { PathDistance }(f i, f j)
$$

$\overline{\text { Size }(\text { FileSets }) \times(\text { Size }(\text { FileSets })-1)}$

We get the average path distance for each community and calculate the average path distance inside each community for all communities $\left(D_{i}\right)$. Similarly, we randomly sample a set of files, which has the same size as each community, and formed a random "community". We calculate the average path distance for all random "communities" $\left(D_{r}\right)$. We compared the results in table 2 . As we can see, the average package distance of a community is 4.23 , while the random files' average package distance is 12.66 . Files in the same community tend to be more localized, which means files in the same community are closer to each other in terms of physical or package distances. For example, in figure 1, the average path distance for files in communities is 2.9 , compared to 4.7 for all files.

Summary: Files in the same community are more likely to share the same characteristics and co-change with each other in revision history. These maintenance communities should be treated together in a system's evolution and deserve special attention.

\section{COMMUNITIES EVOLUTION}

D'Ambros et el. [7] states that one view of software evolution is to cover all the work done to update and fix a software system after its first release. In this paper, we also adopt this view and use all types of issues to investigate communities' evolution. We detected maintenance communities over time and compare their differences to monitor their evolution. For example, Avro has 689 issues; we applied $C P M$ to detect communities 689 times in time sequence.
Table 2: Characteristic in vs inter Communities

\begin{tabular}{|l|r|r|r|r|r|}
\hline Project & $W_{c}$ & $W_{i c}$ & $W_{c} / W_{i c}$ & $D_{i}$ & $D_{r}$ \\
\hline Avro & 0.75 & 0.02 & 30.8 & 1.71 & 11.57 \\
\hline Camel & 0.76 & 0.01 & 130.4 & 5.56 & 17.49 \\
\hline Cassandra & 0.86 & 0.11 & 7.8 & 1.56 & 4.75 \\
\hline Cxf & 0.87 & 0.01 & 66.6 & 7.81 & 21.24 \\
\hline Derby & 0.92 & 0.05 & 17.9 & 3.44 & 7.89 \\
\hline Hadoop & 0.89 & 0.01 & 68.4 & 6.25 & 15.35 \\
\hline Hbase & 0.89 & 0.03 & 32.6 & 4.41 & 14.55 \\
\hline Mahout & 0.8 & 0.01 & 63.9 & 4.7 & 13.68 \\
\hline Openjpa & 0.95 & 0.16 & 6.1 & 5.66 & 12.95 \\
\hline Pdfbox & 0.85 & 0.06 & 14.1 & 4.51 & 12.4 \\
\hline Pig & 0.88 & 0.06 & 14.8 & 2.38 & 8.54 \\
\hline Tika & 0.84 & 0.06 & 14.5 & 4.27 & 12.49 \\
\hline Wicket & 0.83 & 0.01 & 100.5 & 4.73 & 16.71 \\
\hline Zookeeper & 0.76 & 0.12 & 6.4 & 2.18 & 7.58 \\
\hline Average & 0.85 & 0.05 & 41.1 & 4.23 & 12.66 \\
\hline
\end{tabular}

A

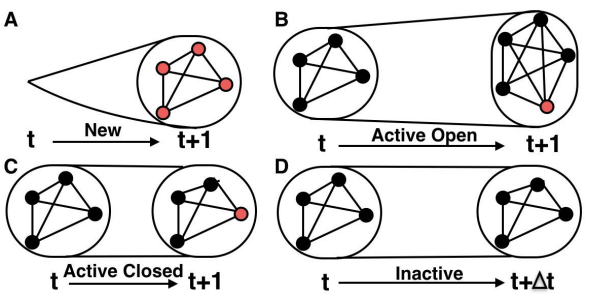

Figure 2: Types of Communities

\subsection{New Communities}

We define that a new community is born at time $t+1$ if at time $t$ it does not exist but at time $t+1$ it is there, as shown in figure 2.A. For example, the issue Wicket-14 modified 3 files (AbstractWebRequestCodingStrategy.java, WebRequestCodingStrategy.java, org.eclipse.core. resources.prefs). These 3 files have not been touched before issue Wicket-14 and thus these files formed a new community.

\subsection{Active Communities}

We define a community as active at time $t+1$ if it meets either one of these two conditions:

1. At time $t$ it exists but at $t+1$ its size is larger than its size at $t$, as shown in figure 2.B. For example, before issue Avro-60, there was a maintenance community consisting of 7 files: (json_tokenizer.h, json.c, avro.h, json.h, lempar.c, json_tokenizer.c and lemon.c). Avro-60 modified 3 files (avro.c, json_tokenizer.c, json.h). avro.c is co-changed with json_tokenizer.c and json. $h$ and joined this maintenance community and became a new member.

2. At time $t$ it existed and at $t+1$ its size is the same as at $t$, and a member in this community is active at time $t+1$, as shown in figure 2.C. For example, before issue Pig-881 on 2009-0710 , there was a community consisting of 3 file members $(M R-$ Compiler.java, POPackage.java and PhysicalOperator.java). Pig-881 touched MRCompiler.java and JarManager.java, in which MRCompiler.java makes this community active at time $t+1$.

\subsection{Inactive Communities}

We consider a community is inactive at time $t+\Delta t$ if this community exists at time $t$ and the files in this community have not been active for a continuous time range (from $t$ to $t+\Delta t$ ). $\Delta t$ is a 
threshold we can set. If we set $\Delta t$ to 100 , this means 100 consecutive issues. For an example, in the Tika project, a 13 member community (MimeTypesFactory.java, PowerPointExtractor.java, WordParser.java, XMLParser.java, OpenOfficeParser.java, ExcelParser.java, PowerPointParser.java, AppendableAdaptor.java, Word6Extractor.java, OfficeParser.java, WordTextBuffer.java, CauseIOException.java and PDF2XHTML.java) has not been active since the issue Tika-186 on 2009-02-14 to 2017, a span of about 940 issues.

\subsection{Reactivated Communities}

If a new member joined in an inactive community or an existing member in this community is active again, we say this community is reactivated. For example, in Cassandra project there is a community consisting of 4 file members (TypeParser.java, CompositeType.java, DynamicCompositeType.java and AbstractCompositeType.java). Since the issue Cassandra-2355 on 2011-05-17, this community has been inactive for over 340 issues. However, on 2012-01-13, the issue Cassandra-3625 modified the file AbstractCompositeType.java and thus this community is deemed to have been reactivated.

\section{RESEARCH QUESTIONS}

RQ1: How many files are clustered into communities? The answer to this question will identify the scope of our study. If files are not in any communities, it means that these files are modified relatively independently in maintenance activities, which suggests good modularization in the system. If a large percentage of maintenance files falls into communities, then these communities should be given higher priority considering their tight bonding.

RQ2: When and why is a new community born? If we can identify when new communities are born, we can alert developers of this new community as soon as it appears. And we are also investigating which types of issues will incur new communities, as a way of getting more insights about new communities.

RQ3: How long can an existing community stay active? An existing active community is a hot maintenance spot in a project. If an active community has been growing and becoming larger, it will cost much more maintenance effort. If files in an existing community keep getting modified, these modified files are hot files, which deserve developers' attention. We are interested in finding the most active communities and investigating how long their activity can last in a project's life.

RQ4: When does a community become inactive? After a period of activity, it is possible that the focus of new development tasks has shifted, or that the old community has been refactored. A community can then be inactive. We are investigating the time when a community becomes inactive.

RQ5: How does an inactive community get reactivated? We are investigating which kinds of issues can reactivate an inactive community. This may help developers find the technical debt that remains in previously inactive communities.

\section{RESULTS}

\subsection{Files in Maintenance Communities}

We have found that the majority of modified files in a project can be clustered into maintenance communities. As shown in figure 3, in a software revision history, there are 3 types of files in the maintenance history after applying CPM: IsolatedFiles, FilesNotInCommunity and CommunityMemberFiles. IsolatedFiles are files which are modified independently in issues; they did not co-change with other files in the revision history. FilesNotInCommunity represents files that do not belong to any community, although they co-change with other files. For example, in figure 1's Avro's communities, file52 and file 53 co-changed with each other, but this does not meet the threshold of 3 to qualify as a community member. CommunityMembersFiles is the set of files in any communities (the colored files in figure 1), which are co-changed with at least two other files in the same community. Figure 3 shows that the majority of all files (74.5\% to $92.5 \%)$ are in maintenance communities during evolution. We can see that Cassandra has the lowest percentage (7.5\%) of IsolatedFiles and FilesNotInCommunity and the highest percentage $(92.5 \%)$ of CommunityMemberFiles, which means that most files in Cassandra are highly coupled in the revision history. This result is consistent with the results in [16], that Cassandra has the lowest decoupling level, a metric which measures to what extent the source files of a system are decoupled.

Answer to RQ1: $74.5 \%$ to $92.5 \%$ of all files in the project revision histories studied can be clustered into communities. Monitoring these non-trivial communities will help developers better access and evaluate the majority of software maintenance activities.

\subsection{The Birth of New Communities}

A new community usually means a shift from old tasks, bugs or issues to new tasks, bugs or issues, which deserves close attention of developers' teams. In this section, we are investigating when and why new communities start to be born.

As we detect new communities continuously, we extract issue information (such as resolution time, issue type, etc.) when a new community is born. Table 3 lists all types of issues that led to a new community. A cell with a gray background means this type of issue is the most in one project. We can see that bug issues causes most new communities, followed by improvement issues and feature issues. If a bug issue causes a new community, either it has new files or its old files have not been touched in the prior revision history and this could cause the birth of a new community. Improvement issues mean refactoring, new classes or files, so it is easy to understand that improvement issues can lead to new communities. When feature issues involve implementation of new classes or files, these issues can lead to new communities as well.

Next, we investigate whether a community's birth time is related to the release time in a project. We extracted all snapshots and tags of release time, as well as the new community formation time, for each project and plotted these in figure 4 . The red circle represents a new community and its birth time and the black circle represents a release/tag time. Figure 4a shows there are no new communities in Avro after Jan. 2014 even though there are releases in Avro, which means that the project has been stable since Jan. 2014. Figure 4c shows that in Hadoop the appearance of new communities slows 


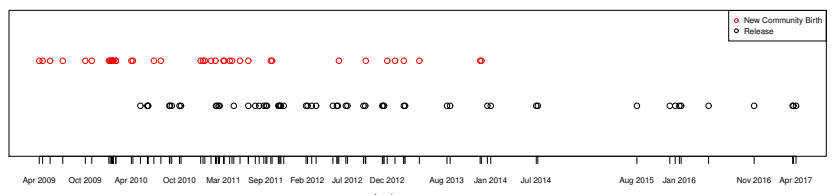

(a) Avro

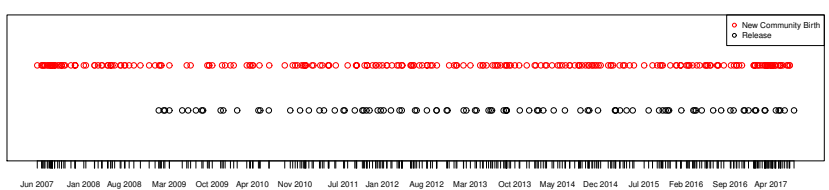

(b) Camel

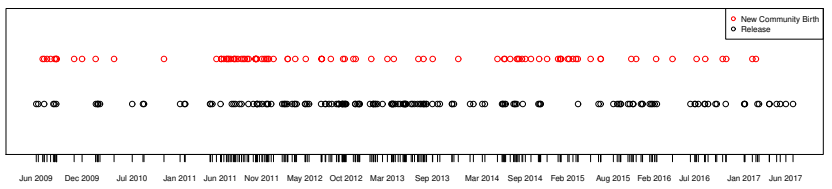

(c) Hadoop

Figure 4: New Communities Form Time vs Tag Time

between May 2012 and March 2014, although there are frequent releases during this time period. In Camel there is continuous creation of new communities, as shown in figure 4b. Overall, we do not see strong correlations between new communities birth time and project release time. This may be because developers in these open source projects are more flexible about new releases and the implementation of these feature/task/bug issues, which will give birth to new communities. We will study commercial systems in the future to see if there is a difference between open source and commercial systems in this regard.

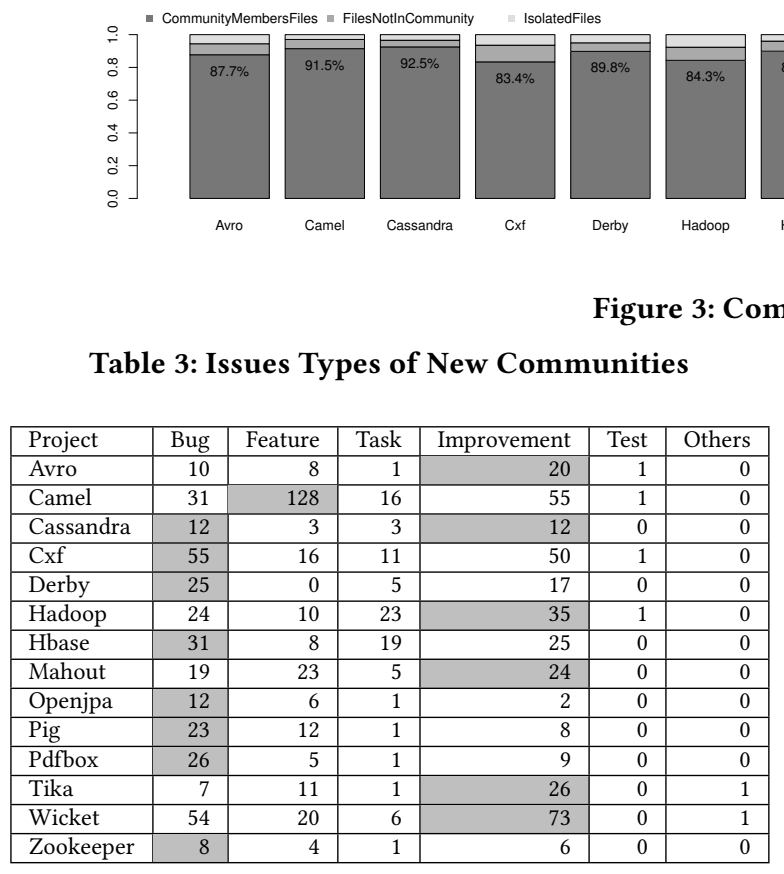

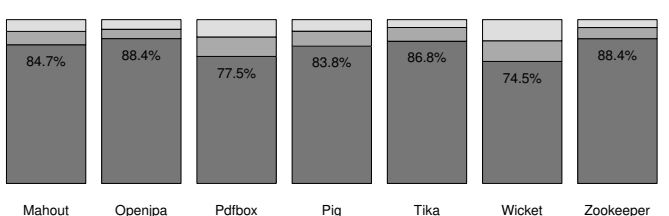

Mahout Openipa Pdrbox
Tika

Zookeeper
Pig

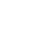

\begin{abstract}
(n)
\end{abstract}

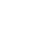

Table 4: Continuous Open(Growing) Communities Steps

\begin{tabular}{|l|r|r|r|r|r|r|}
\hline Project & Step1 & Step2 & Step3 & Step4 & Step5 & Total \\
\hline Avro & 135 & 2 & 1 & 0 & 0 & 138 \\
\hline Camel & 5703 & 571 & 83 & 12 & $2\left(2^{1}\right)$ & 6373 \\
\hline Cassandra & 769 & 99 & 35 & 6 & 0 & 909 \\
\hline Cxf & 2135 & 171 & 18 & 0 & 0 & 2324 \\
\hline Derby & 1089 & 153 & 19 & 3 & 1 & 1265 \\
\hline Hadoop & 312 & 23 & 5 & 1 & 0 & 341 \\
\hline Hbase & 6808 & 992 & 186 & 40 & 1 & 8027 \\
\hline Mahout & 117 & 5 & 0 & 0 & 0 & 122 \\
\hline Openjpa & 212 & 26 & 2 & 2 & 0 & 242 \\
\hline Pdfbox & 211 & 15 & 0 & 0 & 0 & 226 \\
\hline Pig & 1061 & 105 & 11 & 0 & 1 & 1178 \\
\hline Tika & 141 & 11 & 0 & 1 & 0 & 153 \\
\hline Wicket & 318 & 8 & 0 & 0 & 0 & 326 \\
\hline Zookeeper & 89 & 7 & 1 & 1 & 0 & 98 \\
\hline
\end{tabular}

Answer to RQ2: New communities are formed by bug, improvement and feature Issues. In open-source projects, new communities seems unrelated with planned releases.

\subsection{Active Communities}

In this section, we investigate 2 types of active communities. One is active open communities, in which new members keep join in. The other is active closed communities in which old members stay active in revision history.

6.3.1 Active Open (Growing) Communities. If a new member joins a community, we define it is an active open/growing community. If a community is growing continuously over time, we define it as a continuous active open community. The continuous active communities we detected are shown in table 4 . The number of steps means how many issues a community has continued to attract new members in a time series. Figure 5 shows a continuous 4-step active open community in Hadoop. The first community at time 356 contains 8 files. At time 357, issue hadoop-7250 adds file 243 to this community and now it contains 9 files. At time 358 and time 359, file 244 and file 245 are added. Finally at time 360 , this community has 14 files by adding file231, file 246 and file 49 . The longest active open community we have observed has grown over 6 steps. From table 4 we can see most of the continuous active open communities grow in one step but do not continue growing in multiple steps. Only 5 communities in 14 open-source projects have grown over 5 steps and only 2 communities in the Camel project have grown over 6 steps or more.

These continuous active open communities are not mutually exclusive; for example, a continuous active open community may stop growing for a period of time but later will grow again. Our

${ }^{1}$ There are 2 communities with 6 or more steps. 


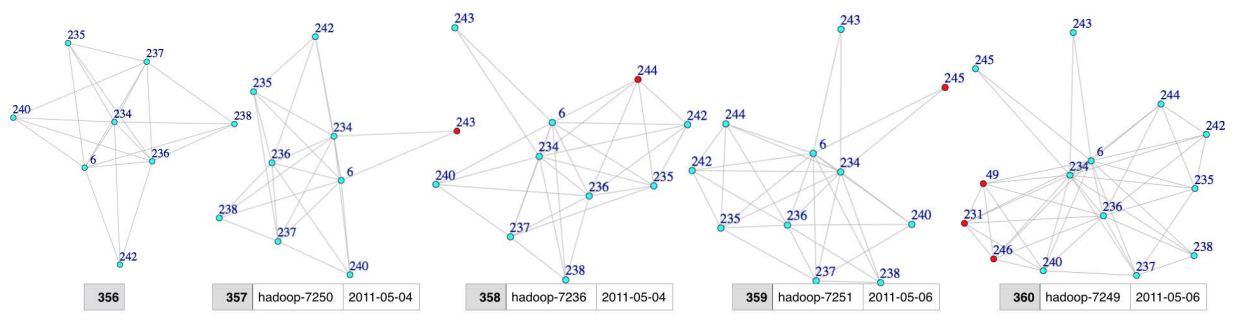

Figure 5: A 4-Step Active Open(Growing) Community in Hadoop

Table 5: Merged Active Open(Growing) Communities

\begin{tabular}{|l|r|r|r|r|}
\hline Project & Count & MaxTimeRange & MaxIncreasedSize & MaxSteps \\
\hline Avro & 49 & 424 & 119 & 26 \\
\hline Camel & 6017 & 4564 & 2490 & 180 \\
\hline Cassandra & 338 & 5011 & 1577 & 674 \\
\hline Cxf & 2034 & 3218 & 1195 & 149 \\
\hline Derby & 1065 & 2049 & 934 & 152 \\
\hline Hadoop & 96 & 1927 & 617 & 140 \\
\hline Hbase & 7583 & 3877 & 2349 & 433 \\
\hline Mahout & 50 & 599 & 153 & 19 \\
\hline Openjpa & 99 & 1164 & 500 & 145 \\
\hline Pdfbox & 72 & 1594 & 486 & 122 \\
\hline Pig & 1089 & 818 & 536 & 55 \\
\hline Tika & 44 & 1009 & 361 & 73 \\
\hline Wicket & 138 & 2420 & 615 & 88 \\
\hline Zookeeper & 15 & 736 & 203 & 89 \\
\hline
\end{tabular}

algorithm detects these as two separate continuous active open communities. We merge such communities if the latter community contains the last member of the prior continuous active open community. Table 5 shows the statistics of merged active open communities. As we can see from table 4, there are 138 continuous active open communities in Avro. These can be merged into 49 communities, as shown in table 5. Multiple continuous active open communities can be merged into one merged community. For example, 98 continuous active open communities can be merged into 15 communities in Zookeeper, which means that on average 6.5 communities were merged together. There are some projects where continuous active open communities are more separate. For example 6373 continuous active open communities can be merged into 6017 active open communities in Camel; thus, on average, 1.1 continuous active open communities were merged together. Compared to Zookeeper, the active growing communities are more isolated or random in Camel.

Table 5 also presents the MaxTimeRange, MaxIncreasedSize and MaxSteps of a merged active open community. MaxTimeRange and MaxIncreasedSize measure the maximum time and the maximum increased number of files, when a merged active open community starts and ends growing (although during some time periods this community's size may remain the same). MaxSteps measures the maximum steps when a merged active open community continuously grows. We can see from table 5 that the MaxIncreasedSize ranges from 119 in Avro to 2490 in Camel. The MaxTimeRange ranges from 424 in Avro to 5011 in Cassandra, which is almost 90\% of its life time. The MaxSteps ranges from 19 in Mahout to 674 in Cassandra, which excludes the time period when a community's size remains unchanged.

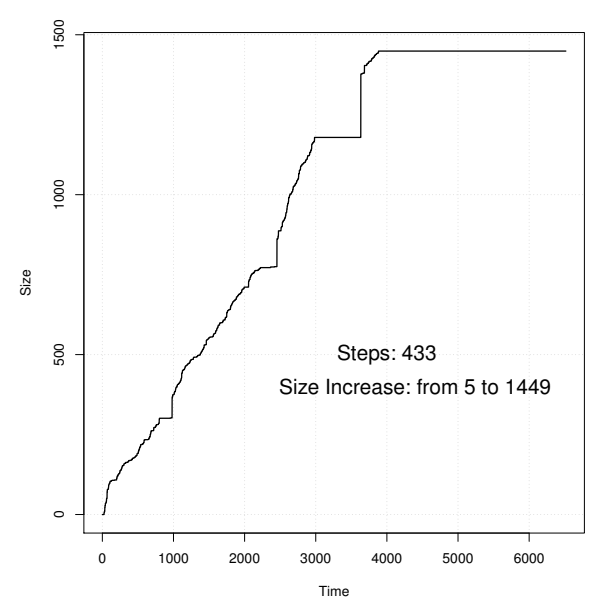

Figure 6: A Merged Active Open Community in Hbase

Figure 6 shows an example of merged active growing communities in Hbase. As we can see, this community starts at the early stage of the project and continues to grow to time stamp 3882. The size of this community has increased from 5 to 1449 files. But the community stop growing after time stamp 3883, which means this community has achieved a temporary saturation and became stable. The long life span and large size of this community makes it a hot spot attracting new members from 433 issues and thus deserves close examination.

6.3.2 Active Closed Communities. If any member in an existing community continues to be changed but the community is not adding new members, it is an active closed community. As with continuous active open communities, most closed communities can stay active continuously in one or two steps as shown in table 6. Some active closed communities even make it to 5 steps. For example, Figure 7 shows an example of active closed community with 5 steps in Pdfbox. From time 39 to time 43, the community's size (20) is unchanged, but at every time step some files in this community had been modified. Compared to active open communities, we have observed some closed communities staying active a little longer. For example, in Cassandra, 173 active closed communities can stay active in 6 or more steps, compared to zero active growing communities with 5 steps or more.

As with merged active open communities, we merged continuous active closed communities if the files in the first step of the latter community contains the files in the last step of a previous 


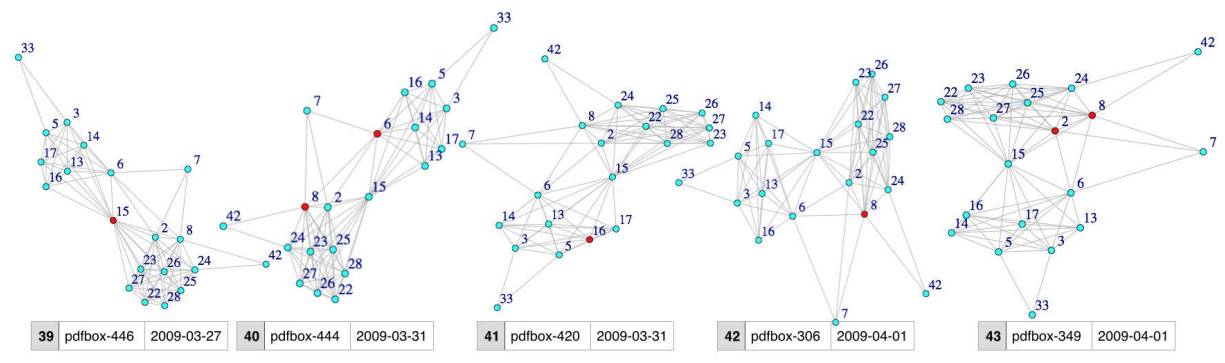

Figure 7: A 5-Step Active Closed Community in Pdfbox

Table 6: Continuous Closed Communities Steps

\begin{tabular}{|l|r|r|r|r|r|r|r|}
\hline Project & Step1 & Step2 & Step3 & Step4 & Step5 & Step6+ & Total \\
\hline Avro & 299 & 35 & 10 & 1 & 1 & 0 & 346 \\
\hline Camel & 2476 & 393 & 163 & 83 & 50 & 34 & 3199 \\
\hline Cassandra & 534 & 218 & 139 & 113 & 76 & 173 & 1253 \\
\hline Cxf & 1574 & 156 & 45 & 15 & 4 & 1 & 1795 \\
\hline Hadoop & 1144 & 101 & 14 & 6 & 2 & 2 & 1269 \\
\hline Hbase & 1381 & 337 & 211 & 109 & 80 & 91 & 2209 \\
\hline Pdfbox & 654 & 94 & 19 & 12 & 4 & 3 & 786 \\
\hline Wicket & 1162 & 72 & 8 & 1 & 0 & 0 & 1243 \\
\hline Derby & 693 & 113 & 48 & 27 & 16 & 22 & 919 \\
\hline Mahout & 310 & 13 & 1 & 0 & 0 & 0 & 324 \\
\hline Openjpa & 221 & 54 & 35 & 21 & 12 & 20 & 363 \\
\hline Pig & 605 & 94 & 47 & 13 & 6 & 6 & 771 \\
\hline Tika & 432 & 60 & 20 & 10 & 5 & 4 & 531 \\
\hline Zookeeper & 172 & 44 & 22 & 22 & 11 & 14 & 285 \\
\hline
\end{tabular}

Table 7: Merged Active Closed Communities

\begin{tabular}{|l|r|r|r|}
\hline Project & Count & MaxTime & MaxStep \\
\hline Avro & 140 & 456 & 27 \\
\hline Camel & 1212 & 5763 & 89 \\
\hline Cassandra & 680 & 4848 & 131 \\
\hline Cxf & 587 & 3070 & 38 \\
\hline Derby & 378 & 1770 & 104 \\
\hline Hadoop & 375 & 1788 & 59 \\
\hline Hbase & 1049 & 4215 & 224 \\
\hline Mahout & 156 & 570 & 19 \\
\hline Openjpa & 192 & 1042 & 43 \\
\hline Pdfbox & 241 & 1476 & 43 \\
\hline Pig & 258 & 1490 & 62 \\
\hline Tika & 165 & 825 & 26 \\
\hline Wicket & 463 & 2393 & 40 \\
\hline Zookeeper & 109 & 526 & 31 \\
\hline
\end{tabular}

community. Table 7 shows the merged active closed communities. There is no MaxIncreasedSize in this table as the size of an active closed community remains the same. The MaxTimeRange is from 456 - 689 in Avro to 5763 - 6396 in Camel. The MaxSteps of active closed community ranges from 19 in Mahout to 224 in Hbase, which is a little smaller than that in merged active open communities.

Figure 8 shows an example of merged active closed communities in Cassandra. As we can see, this community starts at the early stage of the project and continues to be active till the day our data was extracted. This community's size has remained the same: 4 (concurrent/DebuggableThreadPoolExecutor.java, tools/NodeProbe.java, concurrent/IExecutorMBean.java and db/CommitLogExecutorService.java). 131 steps means that the files in this community have been modified in 131 issues, which makes this community a persistent maintenance hot spot and thus deserves close examination.

Answer to RQ3: Active open communities can keep growing continuously at most 6 steps while active closed communities can

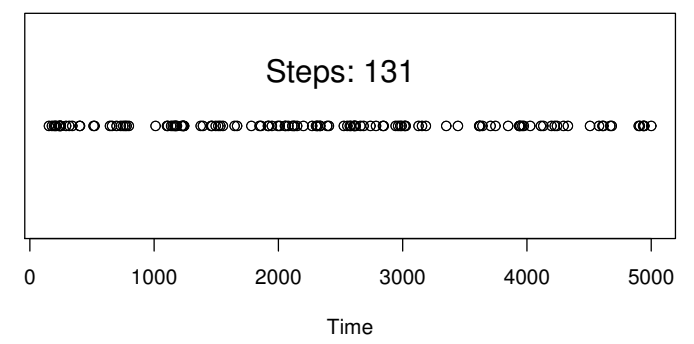

Figure 8: A Merged Active Closed Community in Cassandra

stay active more than 6 steps. Merged active open/closed communities can spread over the entire project history, get involved in hundreds of issues, cost a lot of maintenance efforts, and deserve developers' most attention.

\subsection{Inactive Communities}

If, during a period of time $\Delta t$, existing community members are not active and there are no files joining this community, we define this as an inactive community. If we set $\Delta t$ to $10 \%$, an inactive community is a community which has no activities in at least $10 \%$ of a project's time range. For example, given that Cassandra has 5018 issues and hence a 5018 time span, a community that has no activity in a time span of 502 or more is considered an inactive community. We are interested in when a community starts to be inactive. Figure 9 shows the distribution of inactive communities by a Box-and-Whisker plot. The y axis is the ratio of start time of inactive communities to the entire project's length. We can see that a community in some projects, such as Avro, Hbase and Wicket, can become inactive any time in a project's life, from the beginning to the last time stamp. In some other projects, such as Cassandra, Derby and OpenJPA, inactive communities usually happen at the beginning of the project. As shown in Figure 9, in general, 50\% of existing communities have been inactive at the beginning of a project's development history. This may due to the fast and unstable nature of development in the early stage of a project. Figure 10 shows the CDF graph of inactive community file sizes in Avro, Cassandra and Wicket. As we can see from Figure 10, around $95 \%$ of inactive communities in Cassandra and Wicket and $86 \%$ of inactive communities in Avro have fewer than 10 files. These small inactive communities no longer require developer attention; either they are fixed and have become modularized or the function of these 


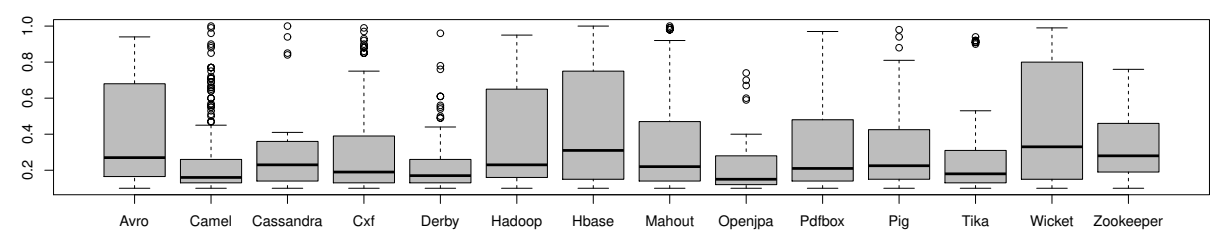

Figure 9: Inactive Communities Starting Time

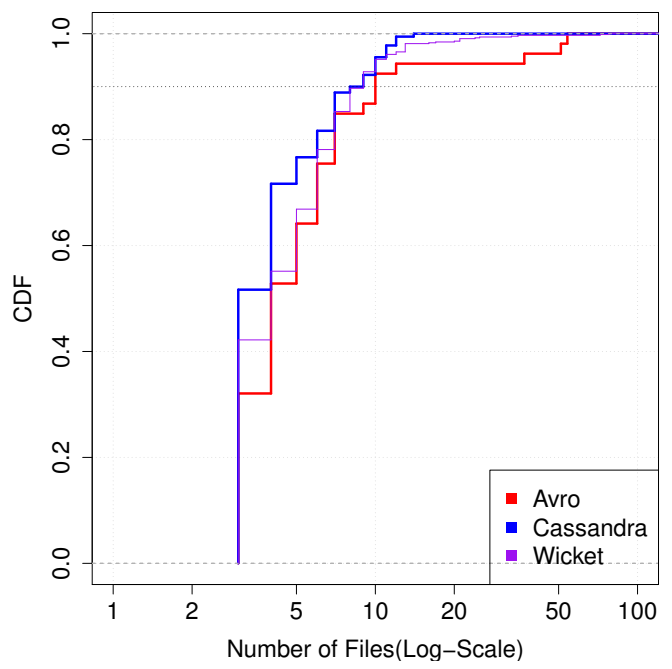

Figure 10: CDFs of the \# of Files in Inactive Communities

Table 8: Statistics of Inactive and Reactivated Communities

\begin{tabular}{|l|r|r|r|}
\hline Project & \#TotalInactive & \#Reactivated & $\%$ \\
\hline Avro & 67 & 23 & $34.3 \%$ \\
\hline Camel & 306 & 187 & $61.1 \%$ \\
\hline Cassandra & 23 & 7 & $30.4 \%$ \\
\hline Cxf & 264 & 141 & $53.4 \%$ \\
\hline Derby & 100 & 75 & $75.0 \%$ \\
\hline Hadoop & 153 & 74 & $48.4 \%$ \\
\hline Hbase & 111 & 39 & $35.1 \%$ \\
\hline Mahout & 166 & 83 & $50.0 \%$ \\
\hline Openjpa & 45 & 37 & $82.2 \%$ \\
\hline Pdfbox & 77 & 46 & $59.7 \%$ \\
\hline Pig & 68 & 41 & $60.3 \%$ \\
\hline Tika & 55 & 23 & $41.8 \%$ \\
\hline Wicket & 281 & 103 & $36.7 \%$ \\
\hline Zookeeper & 33 & 20 & $60.6 \%$ \\
\hline
\end{tabular}

modules have shifted. Such inactive communities can "sleep" for a long time. For example, among 67 inactive communities in Avro, 44 have stayed inactive to the end of the project data (i.e., when our data was extracted).

Answer to RQ4: Existing communities can become inactive during any stage of a project. Half of the inactive communities start at the early stage of development. These inactive communities are usually small.
Table 9: Issue Types of Reactivated Communities

\begin{tabular}{|l|r|r|r|r|r|r|r|}
\hline Project & Bug & Feature & Task & Improvement & Test & Others & Total \\
\hline Avro & 12 & 2 & 2 & 7 & 0 & 0 & 23 \\
\hline Camel & 63 & 23 & 3 & 79 & 1 & 0 & 187 \\
\hline Cassandra & 3 & 0 & 0 & 4 & 0 & 0 & 7 \\
\hline Cxf & 87 & 4 & 3 & 39 & 0 & 2 & 141 \\
\hline Derby & 52 & 0 & 4 & 18 & 0 & 0 & 75 \\
\hline Hadoop & 38 & 4 & 7 & 24 & 0 & 0 & 74 \\
\hline Hbase & 15 & 1 & 4 & 16 & 0 & 0 & 39 \\
\hline Mahout & 47 & 4 & 2 & 30 & 0 & 0 & 83 \\
\hline Openjpa & 23 & 0 & 4 & 8 & 0 & 1 & 37 \\
\hline Pdfbox & 32 & 0 & 1 & 13 & 0 & 0 & 46 \\
\hline Pig & 29 & 1 & 1 & 10 & 0 & 0 & 41 \\
\hline Tika & 9 & 2 & 3 & 9 & 0 & 0 & 23 \\
\hline Wicket & 52 & 7 & 3 & 41 & 0 & 0 & 103 \\
\hline Zookeeper & 9 & 3 & 1 & 6 & 0 & 0 & 20 \\
\hline
\end{tabular}

\subsection{Reactivated Communities}

Given our definition of inactive communities, at a threshold $\Delta t$, provided in the previous section, we now investigate whether an inactive community can become active. That is to say, we are looking into whether files in this inactive community may get modified or a new file is added to this community after a long time of inactivity: more than $10 \%$ of a project's time span. We define such communities as reactivated communities. We first look into how many inactive communities have become reactivated. Then we examine which types of issues can turn an inactive community into a reactivated community.

As shown in table $8,30.4 \%-82.2 \%$ of inactive communities can become reactivated. These reactivated communities can be considered as prior technical debt, which has not been resolved and has incurred additional maintenance costs. The issues which reactivate communities are presented in Table 9. Different from the observation that new communities are initiated by bug and improvement issues, we can see most inactive communities are triggered by bug issues. This justifies our assumptions that these reactivated communities may be paying technical debts from a previous stage, through bug fixes.

Answer to RQ5: $30.4 \%-82.2 \%$ of inactive communities in our dataset have been reactivated. Most reactivated communities are initiated by bug issues, which indicates possible technical debt in previous communities.

\section{DISCUSSION}

\subsection{Threats to Validity}

The first threat to validity of our study is that we use threshold 3 in $C P M$ to detect communities. We chose 3 so that a file in each community is co-changed with at least two other files, which is 
a strong connection in the revision history. Also, we chose $\Delta t$ to be $10 \%$ of a project life to detect inactive communities and reactivated communities. We will experiment with other thresholds for sensitivity testing in the future.

Another threat is that the accuracy of our study depends on the quality of projects' revision history. Recent studies [3, 14] state that it is possible for one commit to fix multiple bugs, or, for a bug commit to not explicitly link to a bug issue. Our analyses assume that files in fixing an issue are related to each other, though we do remove issues which modified more than 10 source files to reduce noise. We intend to further assess this threat via qualitative analysis.

\subsection{Discussion and Future Work}

In this paper, 14 open source systems have been studied to analyze communities' evolution. In the future, we intend to study more projects and, in particular, more commercial projects to compare the difference of life cycles between open source systems and commercial systems. We suspect that the birth of new communities may follow a clearer pattern in commercial systems as they have more focused and organized management. It is also possible that active growing communities have better control and evaluation in commercial systems.

We also plan to propose a metric to better evaluate the activeness in a community. As a community is growing bigger and bigger, some files in this community may not be active. In this case, these old files need to be re-accessed to be qualified as the community member. We will work on this case qualitatively and propose a reasonable methodology to calculate a community's activeness in the future.

\section{CONCLUSIONS}

In this paper, we have presented our empirical study of maintenance communities detection and evolution. Files in maintenance are not isolated. Instead, the majority of these maintenance files co-change with each other and form maintenance file communities. We studied these communities' birth, growth, death and rejuvenation in 14 open source projects. We found new communities are usually formed by bug and improvement issues. An existing community can stay active through the entire life of a system, either by recruiting new members or by modifying its old members. These active open/closed communities cost a lot of maintenance effort and are spread across the entire project lifetime, affect large numbers of files. For this reason they are hot spots worthy of the most attention from developers. An inactive community, usually containing a small set of files, can start as early as the project's beginning. And an inactive community may be activated again, primarily through bug issues.

\section{REFERENCES}

[1] Flore Barcellini, Françoise Détienne, and Jean-Marie Burkhardt. 2008. User and developer mediation in an Open Source Software community: Boundary spanning through cross participation in online discussions. International fournal of Human-Computer Studies 66, 7 (2008), 558-570.

[2] Flore Barcellini, Françoise Détienne, Jean-Marie Burkhardt, and Warren Sack 2007. A socio-cognitive analysis of online design discussions in an Open Source Software community. Interacting with computers 20, 1 (2007), 141-165.

[3] Mike Barnett, Christian Bird, João Brunet, and Shuvendu K Lahiri. 2015. Helping developers help themselves: Automatic decomposition of code review changesets In Proceedings of the 37th International Conference on Software Engineering-Volume 1. IEEE Press, 134-144.
[4] Christian Bird, David Pattison, Raissa D'Souza, Vladimir Filkov, and Premkumar Devanbu. 2008. Latent social structure in open source projects. In Proceedings of the 16th ACM SIGSOFT International Symposium on Foundations of software engineering. ACM, 24-35.

[5] Theodore Chaikalis and Alexander Chatzigeorgiou. 2015. Forecasting java software evolution trends employing network models. IEEE Transactions on Software Engineering 41, 6 (2015), 582-602.

[6] Ned Chapin, Joanne E Hale, Khaled Md Khan, Juan F Ramil, and Wui-Gee Tan. 2001. Types of software evolution and software maintenance. Fournal of Software: Evolution and Process 13, 1 (2001), 3-30.

[7] Marco DâǍŹAmbros, Harald Gall, Michele Lanza, and Martin Pinzger. 2008. Analysing software repositories to understand software evolution. In Software evolution. Springer, 37-67.

[8] Imre Derényi, Gergely Palla, and Tamás Vicsek. 2005. Clique percolation in random networks. Physical review letters 94, 16 (2005), 160202.

[9] Nicolas Ducheneaut. 2005. Socialization in an open source software community: A socio-technical analysis. Computer Supported Cooperative Work (CSCW) 14, 4 (2005), 323-368.

[10] Yulin Fang and Derrick Neufeld. 2009. Understanding sustained participation in open source software projects. Fournal of Management Information Systems 25, 4 (2009), 9-50.

[11] Michael W Godfrey and Daniel M German. 2008. The past, present, and future of software evolution. In Frontiers of Software Maintenance, 2008. FoSM 2008. IEEE, 129-138.

[12] Jungpil Hahn, Jae Yun Moon, and Chen Zhang. 2008. Emergence of new project teams from open source software developer networks: Impact of prior collaboration ties. Information Systems Research 19, 3 (2008), 369-391.

[13] Brandon Heller, Eli Marschner, Evan Rosenfeld, and Jeffrey Heer. 2011. Visualizing collaboration and influence in the open-source software community. In Proceedings of the 8th working conference on mining software repositories. ACM, 223-226.

[14] Kim Herzig and Andreas Zeller. 2013. The impact of tangled code changes. In Mining Software Repositories (MSR), 2013 10th IEEE Working Conference on. IEEE, 121-130.

[15] Qingtao Jiang, Xin Peng, Hai Wang, Zhenchang Xing, and Wenyun Zhao. 2017. Understanding systematic and collaborative code changes by mining evolutionary trajectory patterns. Fournal of Software: Evolution and Process 29, 3 (2017).

[16] Ran Mo, Yuanfang Cai, Rick Kazman, Lu Xiao, and Qiong Feng. 2016. Decoupling level: a new metric for architectural maintenance complexity. In Proceedings of the 38th International Conference on Software Engineering. ACM, 499-510.

[17] Stas Negara, Mihai Codoban, Danny Dig, and Ralph E Johnson. 2014. Mining fine-grained code changes to detect unknown change patterns. In Proceedings of the 36th International Conference on Software Engineering. ACM, 803-813.

[18] Hoan Anh Nguyen, Anh Tuan Nguyen, Tung Thanh Nguyen, Tien N Nguyen, and Hridesh Rajan. 2013. A study of repetitiveness of code changes in software evolution. In Automated Software Engineering (ASE), 2013 IEEE/ACM 28th International Conference on. IEEE, 180-190.

[19] Gergely Palla, Imre Derényi, Illés Farkas, and Tamás Vicsek. 2005. Uncovering the overlapping community structure of complex networks in nature and society. nature 435, 7043 (2005), 814.

[20] Gregorio Robles, Jesus M Gonzalez-Barahona, Martin Michlmayr, and Juan Jose Amor. 2006. Mining large software compilations over time: another perspective of software evolution. In Proceedings of the 2006 international workshop on Mining software repositories. ACM, 3-9.

[21] Luciana L Silva, Marco Tulio Valente, Marcelo de A Maia, and Nicolas Anquetil. 2015. Developers' perception of co-change patterns: An empirical study. In Software Maintenance and Evolution (ICSME), 2015 IEEE International Conference on. IEEE, 21-30.

[22] Damian Tamburri, Rick Kazman, and Hamed Fahimi. 2016. The Architects Role in Community Shepherding. IEEE Software 33, 6 (Nov./Dec. 2016), 70-79.

[23] Qiang Tu et al. 2000. Evolution in open source software: A case study. In Software Maintenance, 2000. Proceedings. International Conference on. IEEE, 131-142.

[24] Lu Xiao, Yuanfang Cai, and Rick Kazman. 2014. Design Rule Spaces: A New Form of Architecture Insight. In Proc. 36th International Conference on Software Engineering.

[25] Lu Xiao, Yuanfang Cai, Rick Kazman, Ran Mo, and Qiong Feng. 2016. Identifying and quantifying architectural debt. In Proc. 38th International Conference on Software Engineering.

[26] Olaf Zimmermann. 2015. Architectural refactoring: A task-centric view on software evolution. IEEE Software 32, 2 (2015), 26-29. 